\title{
A bottom-up pattern collapse mitigation strategy for EUV lithography
}

\author{
Tero S. Kulmala ${ }^{\text {a }}$, Michaela Vockenhuber ${ }^{\text {a }}$, Yasin Ekinci ${ }^{\text {a }}$ \\ ${ }^{a}$ Paul Scherrer Institute, 5232 Villigen PSI, Switzerland, \\ *tero.kulmala@psi.ch, phone +4156310 4573
}

\begin{abstract}
As the lithographic resolution in semiconductor device manufacturing increases photoresist thickness cannot keep the same pace because of limitations set by pattern transfer. This leads to an increase in aspect ratios of patterned resist structures which in turn gives rise to pattern collapse that prevents the use of the patterned features for pattern transfer. Pattern collapse is caused by the capillary forces present on the resist surface during drying of the wafer. Therefore the best approach for mitigating pattern collapse is the complete removal of any drying steps from the processing of wafers after the lithography has been carried out. Several techniques achieving this have been presented. In this paper we propose a bottom-up strategy for pattern collapse mitigation where the wafers are brought from rinsing to further processing steps while they are still wet, thus avoiding the drying-induced pattern collapse without introducing additional processing steps.
\end{abstract}

Keywords: EUV lithography, pattern collapse, resist, development, pattern transfer

\section{INTRODUCTION}

The resolution of lithographically patterned features has been in steady increase over the last decades and this trend is expected to continue in the future [1]. However, the thickness of the resist used for patterning hasn't scaled down at a similar pace. The main reasons for this are that A) the resist structures have to be thick enough for pattern transfer via dry etching or lift-off processes, and B) decreasing the resist thickness leads to increased line-edge roughness (LER) [24]. This trend has resulted in an increase of the aspect ratio $A$ (or, the height of features divided by their width) of the structures patterned in the resist. However, as the aspect ratio increases, pattern collapse becomes a severe challenge. The root cause for pattern collapse are the capillary forces present at the resist surface when the wafer is dried after the resist developing and rinsing processes [5]. This may occur either due to bending or breaking of the resist or its delamination from the substrate.

The maximum stress experienced by the resist due to the capillary forces during the drying process $\sigma_{\max }$ can be expressed as

$$
\sigma_{\max }=\frac{6 \gamma A^{2}}{D \cos \theta}
$$

where $\gamma$ is the surface tension of the rinse liquid, $D$ the spacing between the resist features and $\theta$ the contact angle between the resist and the rinse liquid [5]. For chemically amplified resists (CARs), pattern collapse has been found to cause significant problems at aspect ratios higher than about three and therefore at typical minimum resist thicknesses of around $30-50 \mathrm{~nm}$ the minimum linewidths are limited to about $10-15 \mathrm{~nm}$ when no pattern collapse mitigation is applied.

Several approaches for mitigating pattern collapse in high-resolution patterning have been developed and many of these can be directly inferred from Equation 1. For instance, choosing a rinse liquid - or introducing an additional rinse step with another liquid - with smaller surface tension than that of deionized water, which is the standard rinse material. This approach has been studied in several reports [6-7] and found to mitigate pattern collapse to a limited extent. Furthermore, the optimization of the adhesion between the resist and the surface of the wafer is very important as delamination of the

Extreme Ultraviolet (EUV) Lithography VII, edited by Eric M. Panning, Kenneth A. Goldberg. Proc. of SPIE Vol. 9776, 97762N · @ 2016 SPIE · CCC code: 0277-786X/16/\$18 · doi: 10.1117/12.2219735 
resist is an important factor leading to pattern collapse in resists [4-5]. Therefore, in most cases, the wafers are pretreated or primed prior to applying the resist in order to ensure an optimal adhesion. However, even optimal adhesion cannot prevent the resist lines from bending and touching each other due to the significant capillary forces.

As the capillary forces that occur when the wafers are dried are the root cause for pattern collapse, the most efficient way to mitigate pattern collapse is to eliminate any wafer processing steps where liquid-air interface reaches the resist surface. The most effective technique is critical point (or supercritical) drying [8, 9], where the developed samples are brought, still immersed in a liquid, to a chamber where (typically) liquid carbon dioxide $\left(\mathrm{CO}_{2}\right)$ is added. $\mathrm{Next}$, the temperature and pressure inside the chamber are slowly increased above the so-called supercritical point of $\mathrm{CO}_{2}$, where the densities of its gaseous and liquid state are equal. Above the critical point, the pressure (and the $\left.\mathrm{CO}_{2}\right)$ from the chamber can be slowly released leaving behind the dry sample. As the process involves no formation of a liquid-gas interface, no capillary forces are present, and therefore this technique has been found to be very effective in mitigating pattern collapse in high aspect ratio resist structures [10]. However, critical point drying is a very slow technique, making it incompatible with high-volume manufacturing.

Another technique for removal of the capillary forces, "dry develop rinse process" (DDRP), has been introduced recently [11]. The technique has been proven to be effective in mitigating pattern collapse while being compatible with highvolume manufacturing. In DDRP, the wafer is first exposed, developed, and rinsed as usual (Fig. 1a, i). After rinsing, however, the wafer is not dried but an encapsulating material (a dry develop rinse material, DDRM - typically a siliconbased polymer) is dispensed and spin-coated on the wet wafer (Fig. 1a, ii). The wafer is then baked to remove any residual solvents in the encapsulating material. Next, this layer is etched by an optimized dry-etching process until the top of the buried resist structures is reached (etch-back) (Fig. 1a, iii), followed by another dry-etch step where the resist layer is removed (Fig. 1a, iv), leaving behind only the DDRM pattern which is used in further processing steps. In the process, the wafer is not dried at any point and hence no capillary forces act on the resist structures. Therefore, the root cause for pattern collapse is eliminated. The use of DDRP for organic resists requires extensive optimization of two dryetch processes and results in image reversal of the original resist pattern. In our experiments on DDRP carried out at PSI we have confirmed that pattern collapse can be significantly mitigated using this technique as compared to samples developed using the standard process as shown in Fig. 1b [12].

Sakamoto et al. applied the DDRP process to organic resists [11], which are the workhorse of current high-volume electronics manufacturing. While organic resists, especially CARs, are under further development for use in future semiconductor fabrication nodes $[4,13]$ inorganic resists have recently emerged as a realistic alternative for high-volume manufacturing due to their improved sensitivity, resolution, line-edge roughness, and high etch-resistance [14-15]. We have recently demonstrated a pattern collapse mitigation strategy for inorganic resists inspired by DDRP [16], i.e. the polymer freeze technique. In this method, the photoresist is first exposed, developed, and rinsed as usual (Fig. 1c, i) after which the wafer is not dried but an encapsulating polymer is dispensed and spin-coated on it while it is still wet (Fig. 1c, iii), burying the resist structures in polymer matrix (e.g. PMMA). An additional rinse step can be added (Fig. 1c, ii) to ensure good mixing of the rinse liquid and the encapsulating material. After spin-coating, the wafer can be baked to remove any residual solvents if necessary. Finally, the encapsulating material is removed in a single dry-etch step (Fig. 1c, iv), leaving behind only the original inorganic resist structures. Note that, due to the higher etch-resistivity of inorganic resists, the process is simpler and more straightforward than DDRP, since the process involves only one simple dry-etching step for removing the polymer (i.e. oxygen plasma). Furthermore, no tone-reversal occurs as the original patterned resist structures are preserved. Fig. 1d shows two samples of hydrogen silsesquioxane (HSQ) patterned at 25 $\mathrm{nm} \mathrm{HP}$ at same EUV-IL exposure doses where the samples have been rinsed and dried by $\mathrm{N}_{2}$ flow (left) and where the polymer freeze process has been applied (right). While the wafer that was processed normally suffers from collapsed features, polymer freeze is found to remove the issue in these conditions.

In this paper, we present a novel bottom-up approach to pattern collapse mitigation where the wafers are taken to a subsequent wet processing step (here we have used electroplating as a proof-of-principle demonstration) while they are still wet from the rinse liquid. As the wafers are not dried in between the processes, no capillary forces act on the mechanically weak resist patterns with limited adhesion to the surface and therefore pattern collapse is substantially mitigated and not observed within the range of interest. Here we show how this technique can be applied to transfer linespace (L/S) patterns down to sub-20 nm HP. 


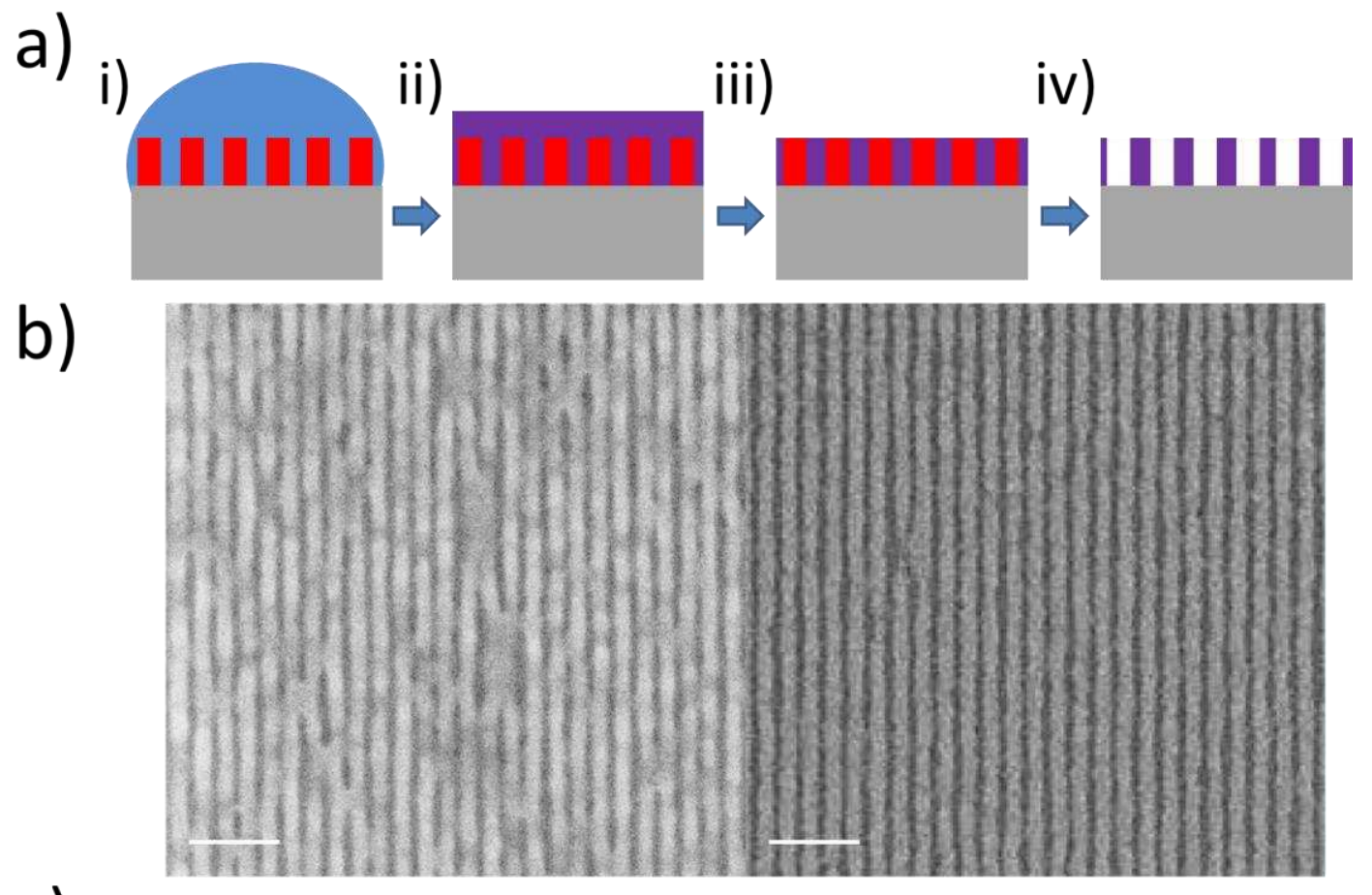

c)
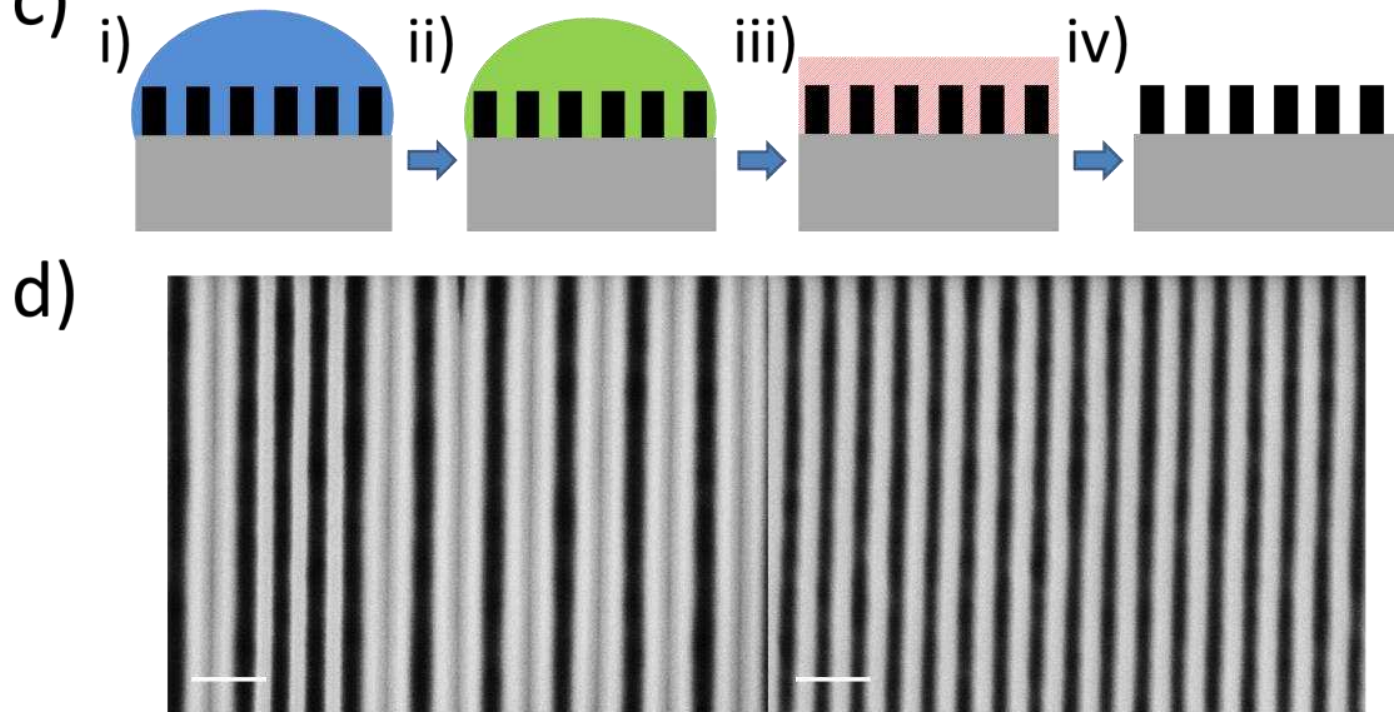

Figure 1 Top-down pattern collapse mitigation strategies. a) In the dry develop rinse process (DDRP) the wafers with organic resist are first exposed, developed and rinsed in deionized water as in a standard process (i), then brought still wet to a spin-coater where an encapsulating material is spin-coated on them (ii), the resist top-level is then exposed in an dry etching step (etch-back) (iii) followed by removal of the resist in another dry etching step (iv). The result is a negative image of the original resist pattern. b) A comparison of wafers with $12 \mathrm{~nm} \mathrm{HP} \mathrm{L/S} \mathrm{patterns} \mathrm{in} \mathrm{an} \mathrm{organic} \mathrm{resist} \mathrm{when} \mathrm{they} \mathrm{have} \mathrm{been} \mathrm{developed,} \mathrm{rinsed} \mathrm{and} \mathrm{dried} \mathrm{normally} \mathrm{(left)} \mathrm{and} \mathrm{when}$ DDRP process has been applied (right). DDRP was found to significantly reduce pattern collapse. c) In polymer freeze process, wafers with inorganic resist are exposed, developed and rinsed as in a standard process (i), rinsed in the solvent of the encapsulating polymer (ii), brought wet on a spin-coater where the encapsulating polymer is spin-coated on it (iii). Finally, the encapsulating polymer layer is removed in a dry oxygen plasma etching step (iv). d) A comparison of two samples with $25 \mathrm{~nm} \mathrm{HP}$ L/S patterns with $85 \mathrm{~nm}$ thick hydrogen silsesquioxane (HSQ) patterns when they have been developed, rinsed in deionized water and dried normally (left) and when polymer freeze process has been applied (right). No pattern collapse is observed for the sample where polymer freeze process was applied. 


\section{EXPERIMENTAL DETAILS}

All the lithographic patterning in this paper was carried out using the EUV-IL exposure tool (XIL-II) at Paul Scherrer Institute (Villigen, Switzerland). The tool utilizes EUV light at $13.5 \mathrm{~nm}$ wavelength from an undulator source of synchrotron facility Swiss Light Source (SLS), providing high brightness and high spatial coherence and making the tool well suited for EUV-IL technique. PSI's EUV-IL tool has demonstrated patterning down to $7 \mathrm{~nm}$ half-pitch [17] and very recently down to $6 \mathrm{~nm}$ (see these proceedings), holding the current world record resolution in photolithography. Further details on the XIL beamline are given elsewhere [18]. Most of the wafer processing was carried out on-site in a dedicated cleanroom in connection with the exposure tool.

The transmission gratings (masks) were fabricated by patterning HSQ lines on 100-nm-thick freestanding $\mathrm{SiN}_{\mathrm{x}}$ membranes by electron beam lithography. In order to block the light outside of the area covered by the gratings, a $\sim 500$ $\mathrm{nm}$-thick layer of gold is deposited via electroplating. Further details on the fabrication of the masks are given in Ref. [19]. For such transmission gratings, the pitch $p$ of the pattern on the mask is reduced in the pattern on the wafer, $g$, as follows:

$$
p=\frac{g}{2 m}
$$

where $m$ is the diffraction order [17]. Typically we use first order diffracted beams for patterning, and therefore the mask pattern resolution is halved on the wafer. This simplifies significantly the resolution requirements of the mask fabrication process.

The effective numerical aperture NA for the XIL tool is given by

$$
N A=\frac{\lambda}{4 \times H P}
$$

where $\lambda$ is the wavelength used (here, $13.5 \mathrm{~nm}$ ). The aerial image created by interference lithography is a sinusoidal pattern and is similar to the case of dipole illumination with projection optics with NA given above.

For imaging the samples, a general-purpose scanning electron microscope (SEM) (Carl Zeiss SUPRA 55VP) and an inlens detector were used. We note that as the characterization of the samples was done by a multipurpose SEM, the obtained CD and LER values are subject to change depending on the SEM settings and condition, although every effort was taken to try to keep the settings constant.

The sample fabrication process is described in Fig. 2. First, an electroplating seed layer consisting of $5 \mathrm{~nm} \mathrm{Cr}$ and $15 \mathrm{~nm}$ $\mathrm{Au}$ is deposited on a 4-inch silicon wafer by thermal evaporation (Fig. 1a). Next, the wafer is primed with hexamethyldisilazane (HMDS) in a vacuum priming chamber to ensure a good adhesion of the resist. A standard chemically amplified, positive tone photoresist is spin-coated on the wafer (resist thickness $\sim 30 \mathrm{~nm}$ ) and baked according to manufacturer's instructions. The resist is exposed with several identical dose range patterns by EUV interference lithography and using grating masks with L/S patterns with varying HP. A post-exposure bake is carried out as instructed by the resist manufacturer. Prior to developing, the wafers are cleaved into several samples to allow for preparation of a reference sample.

The wafers are developed for $30 \mathrm{~s}$ using a standard alkaline developer after which they are rinsed in deionized water (DIW) for $30 \mathrm{~s}$ (Fig. 1b). After rinsing the wafers are not dried but taken to a Ni electroplating bath while the resist patterns are still wet from the DIW used for rinsing (Fig. 1c). For the electroplating, a nickel sulphamate electrolyte solution ( $\mathrm{c}=185 \mathrm{~g} / \mathrm{l}, \mathrm{pH}=3.0, \mathrm{~T}=57{ }^{\circ} \mathrm{C}$, supplier: Enthone Inc.) is used. A magnetic stirrer at the bottom of the electroplating vessel serves to maintain an even ion distribution throughout the whole bath and to maximize deposition uniformity. For the Ni deposition, a duty cycle of $1.4 \mathrm{~s} / 0.6 \mathrm{~s}$ and a plating current of $15 \mathrm{~mA}$ were selected based on our prior work [20]. Under these conditions, the $\mathrm{Ni}$ growth rate is approximately $0.5 \mathrm{~nm} / \mathrm{s}$. After the electroplating, the wafers are rinsed by DIW and dried using a nitrogen gun. Finally, the photoresist is completely removed by exposing the sample to mild oxygen $\left(\mathrm{O}_{2}\right)$ plasma (Oxford Instruments Plasmalab 80 RIE; RF power $50 \mathrm{~W}, 250 \mathrm{mTorr}, 20 \mathrm{sccm} \mathrm{O}_{2}$ 
flow, $60 \mathrm{~s}$ etch time) (Fig. 1d). The reference samples are prepared in an identical way as the electroplating samples apart from that they are dried after the develop and rinse steps using a nitrogen gun.

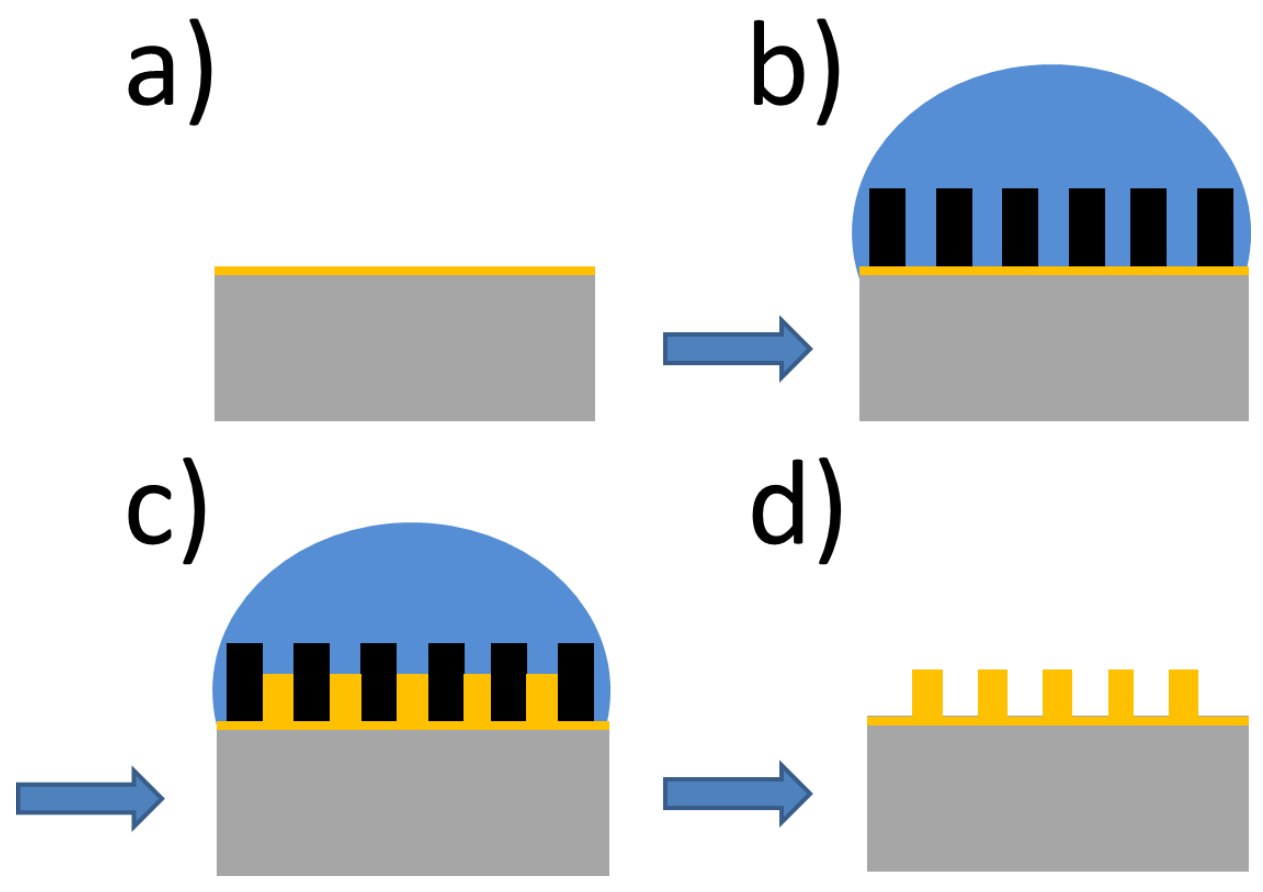

Figure 2. Process flow for fabricating the samples described in this work. a) An electroplating seed layer ( $5 \mathrm{~nm} \mathrm{Cr}+15 \mathrm{~nm}$ $\mathrm{Au}$ ) is evaporated on the wafer. b) A resist is spin-coated, exposed and developed normally. The wafer is rinsed in deionized water. c) The wafer is taken to an electroplating bath while it is still wet from the rinse liquid and a film of $\mathrm{Ni}$ is electroplated in between the resist lines. d) The wafer is rinsed and dried. The resist is removed in mild oxygen plasma, leaving only the electroplated structures on the wafer.

\section{RESULTS}

The results from the experiments are presented in Fig. 3. The top row of Fig. 3 shows scanning electron micrographs of reference samples (that were developed, rinsed and then dried using $\mathrm{N}_{2}$ flow) at HPs of $22 \mathrm{~nm}, 15 \mathrm{~nm}$, and $14 \mathrm{~nm}$. The samples in the bottom row are identical apart from that the Ni electroplating process as explained in the previous section was applied to them. The Ni thickness is approximately $30 \mathrm{~nm}$. From these samples, the resist was removed in a subsequent oxygen plasma etch step as explained above.

As can be seen from the figure, the resist pattern in the reference samples at $22 \mathrm{~nm}$ and $15 \mathrm{~nm}$ HP are very well resolved and free of pattern collapse. Only at $14 \mathrm{~nm}$ HP a partial pattern collapse is observed, yet the resist pattern is still very well resolved. Also, from the $22 \mathrm{~nm}$ HP image it can be observed that the seed layer underneath the resist is not perfectly uniform. This might be due to either roughness or impurities in the metal film or resist residues left over from the resist development process.

The electroplated $22 \mathrm{~nm}$ HP sample shows continuous and relatively uniform Ni L/S pattern. However, at $15 \mathrm{~nm} \mathrm{HP}$ some line breaks are visible yet the lines are still relatively uniform in width. At $14 \mathrm{~nm}$ HP more line breaks are observed and the linewidths are no longer uniform. We believe that the line breaks (at $14 \mathrm{~nm} \& 15 \mathrm{~nm}$ HPs) and the poor uniformity of the pattern (at $14 \mathrm{~nm} \mathrm{HP}$ ) are due to incomplete nucleation of the $\mathrm{Ni}$ on the seed layer caused by either 
impurities on the seed layer, poor transport of $\mathrm{Ni}$ ions onto the wafer surface from the bath solution, or formation of bubbles at the seed layer's surface during the plating process. Nevertheless, our first results are encouraging and further work is ongoing on optimization of the seed layer cleanliness and the electroplating chemistry and parameters.

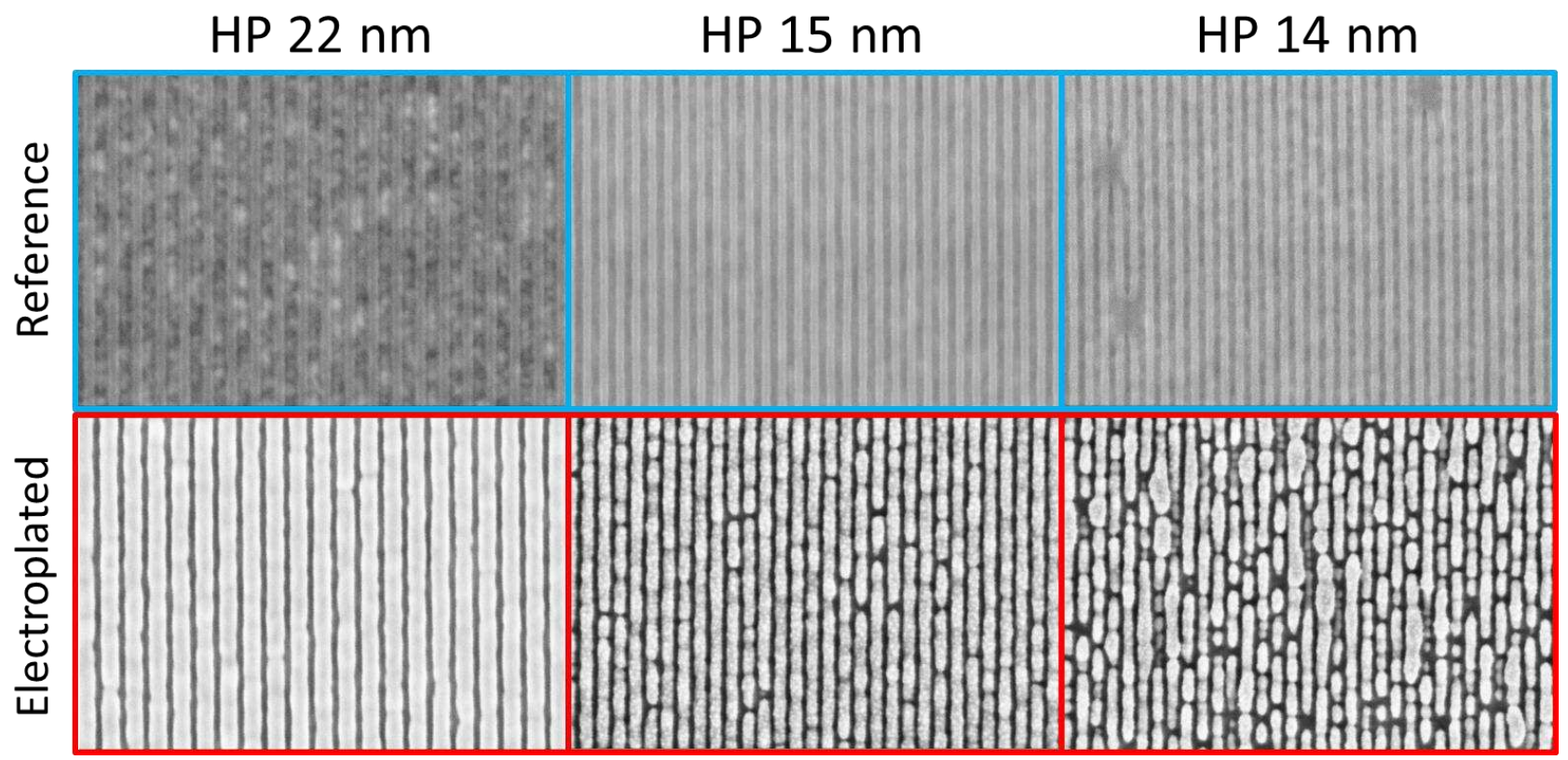

Figure 3 In the top-row, the samples at HPs of $22 \mathrm{~nm}, 15 \mathrm{~nm}$ and $14 \mathrm{~nm}$ have been developed and dried normally. Pattern collapse is observed for the HP $14 \mathrm{~nm}$ sample. The samples in the bottom row were developed and rinsed in an identical process but they were brought directly to a Ni electroplating bath while still wet and $\sim 30 \mathrm{~nm} \mathrm{Ni}$ was electroplated on them. For the two smaller HPs, line breaks and/or non-uniform linewidth are observed. The magnification factor is the same for all the images.

\section{CONCLUSIONS AND OUTLOOK}

Pattern collapse in photoresist structures due to the capillary forces during wafer drying is a potential obstacle for further increase in photoresist resolution. The most common pattern collapse mitigation strategies (reducing resist thickness, optimized resist adhesion, low surface tension rinse liquid, critical point drying) have been employed to their limits and novel and effective techniques are in high demand. Recently, promising results have been achieved by avoiding the drying step in wafer processing by encapsulating the resist structures in a polymer film while they are still wet [11, 16]. However, these top-down techniques increase the number of device fabrication process steps and hence the overall cost. Here, we have proposed a simple, yet novel approach for pattern collapse mitigation where the samples are brought directly from rinsing - while still wet - in the subsequent (wet) process step. As the wafers are not dried, the capillary forces are avoided and the pattern is transferred without collapse-induced defects. Furthermore, in this bottom-up technique, no extra steps are added to the process. This technique can be scalable to high-volume manufacturing and requires no special equipment for its implementation.

We have shown that with this technique it is possible to transfer $22 \mathrm{~nm} \mathrm{HP}$ pattern to Ni and that the results for 14 and 15 $\mathrm{nm}$ HPs also seem promising. We believe that there is plenty of room for improving the results by optimizing the Ni electroplating parameters and chemistry (e.g. by using Ni citrate $\mathrm{C}_{12} \mathrm{H}_{10} \mathrm{Ni}_{3} \mathrm{O}_{14}$ as electrolyte). Furthermore, we intend to introduce mild ultrasonic agitation of the bath during the plating process which we believe will help in removing any gas bubbles that might form at the wafer surface during the plating process hindering Ni growth. Moreover, as the gold we have used in the seed layer is not compatible with high-volume manufacturing, we intend to explore alternative seed layer materials, such as platinum. As the cleanliness of the seed layer is of paramount importance, we will also experiment on different ways of cleaning it prior to the lithography or electroplating steps. 
Here, we have used $\mathrm{Ni}$ electroplating as a proof-of-principle for the technique presented. However, we note that the approach introduced here is combinable with any other wet deposition or etching process. For example, other growth methods resulting in patterns with better mechanical stability or etch resistance can be employed. Alternatively, the resist pattern can be transferred to the substrate by first etching a thin hard-mask layer underneath it by wet etching followed by photoresist removal and dry etching. In general, the concept of pattern transfer without drying the resist pattern has a great potential and should be explored extensively. We would like to address some of the possible methods in the future.

\section{ACKNOWLEDGEMENTS}

The authors would like to thank Dr. James M. Blackwell of Intel Corp. and Mr. Daniel Fan (PSI) for invaluable discussions. We are grateful to Mr. Markus Kropf (PSI) for his technical assistance. Part of this work was performed at the Swiss Light Source, Paul Scherrer Institute.

\section{REFERENCES}

[1] www.itrs.net, visited 4 March 2016.

[2] Singh, L., et al., "Effect of top coat and resist thickness on line-edge roughness," Proc. SPIE 6153, 61530W (2006).

[3] Gallatin, G.M., "Resist blur and line edge roughness," Proc. SPIE 5754, 38 (2005).

[4] Kulmala, T.S., et al., "Toward $10 \mathrm{~nm}$ half-pitch in extreme ultraviolet lithography: results on resist screening and pattern collapse mitigation techniques," J Micro/Nanolith. MEMS/MOEMS 14 (3), 033507 (2015).

[5] Tanaka, T., M. Morigami, and Atoda, N., "Mechanism of resist pattern collapse during development process," Jpn. J. Appl. Phys. 32, 6059 (1993).

[6] Hien, S., et al., "Collapse behavior of single layer 193- and 157-nm resists: use of surfactants in the rinse to realize the sub-130-nm nodes," Proc. SPIE 4690, 254 (2002).

[7] Tanaka, K., et al., "Improvement of pattern collapse issue by additive-added D.I. water rinse process," Proc. SPIE 5039, 1366 (2003).

[8] Tanaka, T., et al., "Freeze-drying process to avoid resist pattern collapse," Jpn. J. Appl. Phys. 32 (12R), 5813 (1993).

[9] Namatsu, H., K. Yamazaki, and K. Kurihara, "Supercritical drying for nanostructure fabrication without pattern collapse," Microelectron. Eng. 46 (1), 129 (1999).

[10] Goldfarb, D.L., et al., "Pattern collapse mitigation strategies for EUV lithography," Proc. SPIE 8322, 832205 (2012).

[11] Sakamoto, R., Y. Sakaida, and B.-C. Ho, "Dry development rinse process (DDRP) and material (DDRM) for novel pattern collapse free process," Proc. SPIE 8682, 868205 (2013).

[12] W. Shibayama, Proceedings of EUVL Symposium 2015, Maastricht (2015).

[13] Buitrago, E., et al., "Evaluation of EUV resist performance using interference lithography," Proc. SPIE 9422, 94221S (2015).

[14] Grenville, A., et al., "Integrated fab process for metal oxide EUV photoresist," Proc. SPIE 9425, 94250S (2015).

[15] Ober, C., et al., "New developments in ligand-stabilized metal oxide nanoparticle photoresists for EUV lithography," Proc. SPIE 9422, 942207 (2015).

[16] Kulmala, T.S., et al., "Pattern collapse mitigation in inorganic resists via a polymer freeze technique," Microelectron. Eng. 155, 39 (2016).

[17] Mojarad, N., et al., "Single-digit-resolution nanopatterning with extreme ultraviolet light for the $2.5 \mathrm{~nm}$ technology node and beyond," Nanoscale 7 (9), 4031 (2015).

[18]Ekinci, Y., Vockenhuber, M., Mojarad, N. and Fan, D., "EUV resists towards 11 nm half-pitch," Proc. SPIE 9048, 904804 (2014).

[19] Mojarad, N. et al., "Broadband interference lithography at extreme ultraviolet and soft x-ray wavelengths," Optics Letters 39, 2286 (2014).

[20] Hili, K. et al., Microelectron. Eng. 141, 122 (2015). 